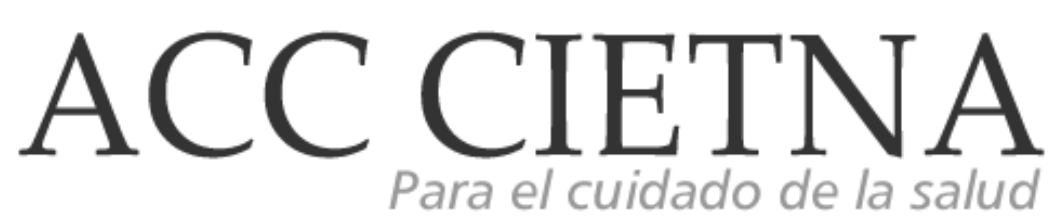

https://doi.org/10.35383/cietna.v3i2.43

\title{
Conocimientos y prácticas sobre Lactancia Materna Exclusiva en madres primíparas que acuden al Servicio de Crecimiento y Desarrollo del Hospital Naylamp, Chiclayo.
}

\author{
Villegas Mera Estela Violeta ${ }^{1}$, Arriola Arízaga María Caridad ${ }^{2}$
}

\begin{tabular}{ll}
\hline INFORMACIÓN DEL ARTíCULO & RESUMEN \\
\hline Historia del artículo: & Este artículo reporta una investigación que tuvo por objetivo determinar \\
Recibido el 23 de mayo de 2016 & el nivel de conocimientos y prácticas sobre lactancia materna exclusiva \\
Aceptado el 10 de octubre de 2016 & en madres primíparas que acuden al servicio de crecimiento y \\
\hline Palabras claves: & desarrollo del Hospital Naylamp. La hipótesis formulada fue considerar \\
Lactancia materna exclusiva & si la muestra en estudio posee un nivel regular de conocimientos y \\
Conocimiento & prácticas sobre Lactancia Materna Exclusiva. Fue una investigación \\
Práctica & cuantitativa , descriptiva, transversal, con una muestra de 127 madres \\
Madre primípara. & primíparas. Los datos del nivel de conocimientos se recogieron con un \\
& cuestionario validado en prueba piloto y del nivel de práctica con lista \\
& de cotejo. Se tuvo en cuenta los principios éticos personalistas y \\
& criterios del rigor científico. Se concluye que el nivel de conocimientos \\
& que tienen las madres primíparas sobre lactancia materna exclusiva, es \\
& regular con $46.5 \%$ el nivel de práctica sobre la técnica de \\
& amamantamiento es bueno con 43.3 \%, aceptándose la hipótesis \\
& formulada.
\end{tabular}

\footnotetext{
${ }^{1}$ Licenciada en Enfermería. Enfermera del Hospital Naylamp - Chiclayo, Perú. Email: estela_1027@hotmail.com
}

${ }^{2}$ Magister en Bioetica. Docente de la Escuela de Enfermería de la Universidad Católica Santo Toribio de Mogrovejo. Licenciada en Enfermería, Jefa 


\section{Knowledge and practices Exclusive breastfeeding in primiparous mothers who come to the Growth and Development Service of Naylamp, Chiclayo Hospital}

\section{ABSTRACT}

Keywords:

Exclusive breastfeeding

knowledge

Practice

Primiparous mother
The present investigation called knowledge and Practices on exclusive Breastfeeding in first-time mothers who go to the Growth and Development of Naylamp Hospital, was to: Determine the level of knowledge and practice exclusive breastfeeding in primaparous mothers who go to the growth and development of Naylamp Hospital, your hypothesis was to consider whether the time mothers attending the service of growth and development Naylamp Hospital have a regular level of knowledge and practice exclusive breastfeeding. It was a quantitative, descriptive, transversal, with a sample of 127 first-time mothers, was considered inclusion criteria: mothers with first child, mothers who provided breast milk only 0-6 months old. To assess the level of knowledge was used as a tool: the questionnaire, validated with a pilot test to 24 first-time mothers, to assess the level of practice we used the instrument: the checklist. Given personalist ethical principles and criteria of scientific rigor. The result was, that the level of knowledge that are first-time mothers on exclusive breastfeeding is regular with $46.5 \%$, the level of practice on breastfeeding technique is good with $43.3 \%$. Finally was concluded that the level of knowledge that are first-time mothers on exclusive breastfeeding is normal, however to evaluate practice on breastfeeding technique was good, agreeing on the one hand with the hypothesis formulated above.

\section{Introducción}

Desde que existe la humanidad, la leche materna constituye la principal fuente de alimento para el niño pequeño. Durante la $55^{\mathrm{a}}$ Asamblea Mundial de Salud, conocida como "Estrategia Mundial para la Alimentación del lactante y del Niño pequeño" realizada en Ginebra, se recomendó que la lactancia materna exclusiva deba realizarse durante los primeros seis mes es de vi da, y continuar posteriormente con alimentos complementarios, hasta los dos años como mínimo. La leche materna es el único alimento capaz de satisfacer todas las necesidades de nutrientes durante los primeros seis meses de vida'. Asimismo, es un alimento único e insustituible que aporta al lactante todos los nutrientes que necesita, en la cantidad y proporción adecuada, durante los 6 primeros meses de vida; y una parte importante de los mismos desde los 6 meses a los 2 años. Está adaptada genéticamente no solo a la especie, sino 
también a cada niño y a sus necesidades y grado de desarrollo.

La leche humana se adapta a las necesidades nutricionales del lactante contiene lípidos que representa su principal fuente de energía, ácidos grasos esenciales para el crecimiento y desarrollo del cerebro, retina y los centros auditivos. La lactosa es el principal hidrato de carbono, necesario para el crecimiento y desarrollo del sistema nervioso central; así mismo las proteínas proporcionan aminoácidos esenciales, que favorecen el desarrollo de este mismo sistema². Además, la cantidad de agua de la leche materna es del $87 \%$, por eso él bebe amamantado no necesita agua complementaria. La leche materna brinda la nutrición ideal, protege contra la infección y la muerte, reduce el riesgo de algunas enfermedades a largo plazo en el niño y en la madre ${ }^{3}$. El vínculo que se establece en la Lactancia Materna Exclusiva, le brinda al bebé una sensación de bienestar y seguridad, lo que constituye la primera experiencia social de su vida, de esta manera le potencializa un autoestima adecuada y una inteligencia emocional para toda la vida ${ }^{4}$.

En el 2001, la OMS en colaboración con UNICEF, emite la recomendación internacional de promover y garantizar condiciones para una lactancia materna exclusiva desde el instante del nacimiento del recién nacido hasta los seis meses de edad. Asimismo en el 2002, se insiste en la lactancia materna exclusiva de los meses antes mencionados y si es posible mantenerla hasta los dos años. Paralelamente y desde hace ya más de treinta años, el Código Internacional de Comercialización de Sucedáneos de la Leche Materna (1981), la Declaración Innocenti sobre la Protección, Promoción y Apoyo a la Lactancia Materna (1990), así como la Iniciativa Hospitales Amigos de la Infancia (1991), han venido llamando la atención de los estados y la opinión pública a nivel mundial sobre los beneficios de la lactancia materna, así como sobre los riesgos y costos en los que incurren familias y estados que no la promueven, la protegen y la difunden ${ }^{5}$.
En Chile, a fines de la década del 70, la lactancia materna exclusiva no superaba el $5 \%$, motivo que se unió al Programa de Fomento Mundial de la Lactancia Materna de UNICEF, denominado "Iniciativa Hospital Amigo del Niño y de la Madre (IHANM) a partir del año 1992, con el objetivo de implementar y evaluar el programa de UNICEF "IHANM", realizándose mecanismos de intervención como: diagnóstico de las prácticas de lactancia, capacitación del personal, cambios administrativos y técnicos en atención del parto y recién nacidos, donde se pondría en práctica el contacto precoz madre hijo y la incorporación del progenitor a sala de partos. Facilitando el contacto precoz, disminución de hospitalización por fototerapia, y los costos de atención del recién nacido, mejorando así la prevalencia de lactancia materna exclusiva al sexto mes de vida.

Evidenciándose entre los años 94 - 97 porcentajes óptimos de $90 \%$ y $80 \%$ en el primer y tercer mes de vida del recién nacido respectivamente', en Brasil en el año 2005 la prevalencia de lactancia materna exclusiva fue de $32.5 \%$.

La OMS y UNICEF, la prevalencia de lactancia materna exclusiva reportada en diferentes estudios es variada, así tenemos que a nivel de Europa; se reporta una prevalencia a los seis meses de Lactancia Materna Exclusiva en el año 1998 de 3.9\%; en Norte América: Estados Unidos, en el 2001, la prevalencia fue de 32.5\%; en México en el 2003, reportó una prevalencia de lactancia materna exclusiva al cuarto mes de $30.5 \%$. Asimismo la lactancia materna exclusiva antes de los seis meses, se registró un incremento significativo de $48.7 \%$ en el 2006 a $53.1 \%$ en el 2010. Con respecto al acumulado de niños con lactancia materna exclusiva a los seis meses, fue de $16.9 \%$ en el 2002 a $21.9 \%$ en el 2006 , mientras que en los últimos cuatro años no ha variado5.

En el año 2010 con datos del primer trimestre informa que las mayoría de las madres alimentan a los menores de seis meses con leche materna siendo mayor en el área rural con $81.2 \%$ que en al 
área urbana 54.6\%. Así mismo dicha proporción ha disminuido cinco puntos porcentuales respecto a la ENDES de 2009 , la cual fue de $68,5 \%$ a $63,2 \%$. Así mismo ENDES 2010, visualizaron a nivel nacional en la región de la costa bajó de 59,3\% a $46,7 \%$ la proporción de menores de seis meses con lactancia materna exclusiva en relación con lo observado en la sierra y en la selva, donde se incrementa de $76,2 \%$ a $80,3 \%$ y de $77,6 \%$ a $78,5 \%$, respectivamente 8 .

A nivel nacional UNICEF, presentó un estudio sobre la situación nutricional referente a la exclusividad de la Lactancia Materna de la niñez en el Perú, donde se evidenció que las cifras sobre la situación de la lactancia materna exclusiva a los niños menores de 6 meses, se observó un ligero incremento de dos puntos porcentuales, al pasar de $67 \%$ en el año 2000 a $69 \%$ en el 2009. Sin embargo a diferencia de otros indicadores, es, en el área urbana donde se muestran las cifras más preocupantes, al registrar en el 2009 solo un $61 \%$ de niños menores de seis meses que recibieron lactancia materna, mientras que en ámbito rural la cifra llega a $83 \%$. Lima metropolitana tuvo el menor porcentaje de lactancia materna con el $57 \%$ (Últimas cifras revelan que en el 2010 en Lima metropolitana el porcentaje de lactancia materna exclusiva bajó a 49,1\%) ${ }^{9}$, además, la mayor proporción de menores de seis meses que se alimentan con lactancia materna exclusiva, se presentan en los departamentos de: Puno (97.3\%), Huancavelica (94.7\%), Junín (91.5\%), Ucayali (87.2\%), Apurímac (86.3\%), Huánuco (83.4\%), Cusco (82.3\%) y Ayacucho con el (82.0\%) ${ }^{10}$.

A nivel local, durante la realización de las prácticas clínicas en el Hospital Naylamp, el cual es un establecimiento de nivel I, en el Servicio de Crecimiento y Desarrollo, se pudo observar a muchas madres esperando turno para ser atendidos por la enfermera, las cuales amamantaban a sus niños, adoptando diferentes posiciones, unas de pie, otras sentadas, dificultando al niño un buen agarre y por lo tanto una técnica de amamantamiento positiva; asimismo se observó que algunas madres primíparas utilizaban biberones con fórmula láctea.

Por esta problemática, se planteó la siguiente interrogante de investigación: ¿Cuáles son los conocimientos y prácticas sobre lactancia materna exclusiva en madres primíparas que acuden al servicio de crecimiento y desarrollo del Hospital Naylamp?, teniendo por objetivo general: Determinar el nivel de conocimientos y prácticas de la lactancia materna exclusiva en las madres primíparas que acuden al servicio de crecimiento y desarrollo del Hospital Naylamp. Y como objetivos específicos: Identificar y analizar el nivel de conocimiento sobre lactancia materna exclusiva que poseen la muestra en estudio así como identificar la técnica correcta de amamantamiento en las madres primíparas que acuden al servicio de crecimiento y desarrollo de la institución antes mencionada.

Se tuvo como hipótesis: Las madres primíparas que acuden al servicio de crecimiento y desarrollo del Hospital Naylamp, posee un nivel regular de conocimientos y practicas sobre lactancia materna exclusiva.

\section{Metodología}

La presente investigación, fue de tipo cuantitativa, descriptivo-transversal ${ }^{11}$, utilizando herramientas estadísticas y muestra representativa. La población estuvo conformado por 240 madres primíparas que acudieron al servicio de crecimiento $y$ desarrollo del Hospital Naylamp. La muestra estuvo conformada por 127 madres, los cuales cumplieron los criterios de inclusión y exclusión, estaban incluidas las madres que tenían hijo por primera vez, madres que amamantaban solo con leche materna. Para determinar esta muestra se utilizó la fórmula estadística de la proporción.

El instrumento cuestionario se validó con una prueba piloto ${ }^{12}$, considerándose el $10 \%$ de la muestra, representado por 24 madres del mismo 
Hospital Naylamp, a quienes se les excluyó de la investigación; presentándose algunos inconvenientes como: la falta de tiempo de las madres para responder a los cuestionarios, las labores domésticas que tenían que realizar en sus hogares, pero fueron superados por las investigadoras, adecuándose al tiempo de las madres.

El instrumento constó de tres partes: en la primera parte se obtuvieron los datos generales de los sujetos de estudio, la segunda parte estuvo dirigida a los conocimientos de la lactancia materna exclusiva, la cual constó de 7 preguntas con respuestas múltiples, la tercera parte se refería al conocimiento de prácticas sobre la técnica de amamantamiento, estuvo conformada de 5 preguntas con respuestas múltiples. En este cuestionario se tuvo en cuenta el siguiente puntaje: de 70 a 100\% para la categoría de bueno; de 40 a $69 \%$ para la categoría de regular y de $<$ de $40 \%$ para la categoría de malo.

Para determinar el nivel de práctica de la lactancia materna, se utilizó como instrumento, una lista de cotejo, que permitió identificar comportamientos con respecto a actitudes, habilidades y destrezas de la técnica de amamantamiento. Se aplicó la técnica de la observación, que permitió percibir la realidad estudiada. El instrumento lista de cotejo constó de cinco indicadores de logro en el que se constató, en un solo momento, la presencia o ausencia de éstos, mediante la actuación del sujeto. Con valores de 5 al 20, y puntajes de 85 a $100 \%$ para la categoría de muy bueno; de 70 a $84 \%$ para la categoría de bueno; de 55 a $69 \%$ para la categoría de regular; de 40 a $54 \%$ para la categoría de malo y $<$ de $40 \%$ para la categoría de muy malo.

La recolección de datos, fue realizada previo permiso de la Dirección del Hospital Naylamp de EsSalud, hospital de Nivel I, con más de 120,000 personas adscritas; coordinándose con las autoridades pertinentes y para aplicar el consentimiento informado. Para la selección de la muestra se tuvo en cuenta los criterios de inclusión: Madres primíparas con hijo de 0 a 6 meses de edad, madres primíparas que brindaban solo lactancia materna; madres que aceptaron la aplicación del cuestionario. Los datos fueron procesados en el programa SPSS.

\section{Resultados, análisis y discusión}

El nivel de conocimientos que tienen las madres sobre lactancia materna exclusiva es regular, con $46,5 \%$, asimismo el $22 \%$ tienen un conocimiento bueno, en cambio el $31.5 \%$ tienen un nivel de conocimiento malo, observándose que las madres primíparas aún desconocen aspectos fundamentales sobre lactancia materna exclusiva. Con respecto al nivel de práctica de la técnica de amamantamiento, se obtuvo que en el $74,8 \%$ presenta un nivel de practica bueno; asimismo, el $22 \%$ el nivel de conocimiento de práctica es regular y un $3,2 \%$ el nivel de conocimiento es malo.

Conocimientos que tienen las madres por la publicidad que ven o escuchan o por los familiares que tienen a su alrededor.

Respecto al nivel de práctica de la lactancia materna se obtuvo: que en el $23,6 \%$ fue muy bueno, el $43,3 \%$ tiene un nivel de prácticas bueno, el $31,5 \%$ regular y el $1,6 \%$ tiene nivel de prácticas malo. Observamos, que aún falta enseñar a las madres primíparas en lo que respecta a la práctica, para obtener una muy buena técnica de amamantamiento.

El presente trabajo de investigación se encontró: Que del total de las madres encuestadas, el 46.5\%, presentan un nivel de conocimientos sobre Lactancia Materna Exclusiva, regular. Según Vargas, el conocimiento es una determinación del sujeto por el objeto, es verdadero si su contenido concuerda con el objeto, esto implica un proceso intelectual de enseñanza y aprendizaje, además que hubieron madres primíparas entre 19 y 25 años, las cuales adquirieron conocimientos por 
medio de otras personas o medios de comunicación; Seguido de un nivel de conocimiento malo, equivalente al $31.5 \%$, quienes no tuvieron ninguna experiencia al respecto y solo un $22 \%$ de madres, evidenciaron tener un nivel de conocimiento, bueno, este grupo de madres conocen todo lo relacionado a la lactancia materna.

Las madres saben instintivamente como amamantar a sus hijos; sin embargo el $34.6 \%$ tienen conocimiento acerca de la importancia de brindarles lactancia materna exclusiva a su niño hasta los seis meses de edad, por ser este, un alimento completo, complejo, e inimitable, que proporciona el aporte nutritivo necesario para el crecimiento y desarrollo del niño. Además tiene grandes beneficios tanto para el niño, la madre, y la sociedad. En primer lugar el $36.2 \%$ respondieron que solo beneficia al niño porque contiene todos los nutrientes indispensables para su alimentación, por lo que mejora el estado nutricional, mayor protección inmunológica, lo que disminuye de forma muy importante los episodios infecciosos durante los primeros meses de vida; menor riesgo de sensibilización alérgica, disminuye la morbilidad y mortalidad infantil, previene las enfermedades posteriores como la diabetes, aterosclerosis, obesidad, hipertensión arterial, tiene un efecto laxante suave que facilita la expulsión de meconio, lo que ayuda a prevenir la acumulación de bilirrubina, menor riesgo de padecer mala oclusión dentaria, favorece el desarrollo visual, mayor desarrollo cognitivo en niños nacidos a término. Asimismo, mediante el calostro que es la primera secreción láctea, le protege contra infecciones, proporciona vitaminas A y le sirve al recién nacido como su primera inmunización, lo cual le hace más resistente a ciertas enfermedades infecciosas como enfermedades respiratorias, gastrointestinales; además de ofrecer un bienestar psicológico y afectivo, e intelectual, que contribuye a la disminución de las enfermedades crónicas. Por lo tanto, las madres manifiestan, que su hijo es lo primero, porque piensan que solo el niño es el beneficiado.

También está claro que con la práctica de la lactancia materna exclusiva, se benefician los dos (madre y niño), ya que el $33.1 \%$ de las madres primíparas respondieron, que dicha práctica fortalece los lazos afectivos entre ellos dos a través del contacto físico, prolonga la duración de la amenorrea inducida por la lactancia, previniendo la aparición de anemia después del parto, disminuye los riesgos de hipertensión y de depresión postparto ${ }^{13}$. Así también disminuye el riesgo de tener en un futuro cáncer de mama, de ovario y de fracturas de cadera, así como también el riesgo de sufrir osteoporosis; asimismo, favorece también la pérdida de peso ganado durante el embarazo ${ }^{12}$. Y solo un mínimo $(2,4 \%)$ respondieron que beneficia solo a la madre. Por tanto, la lactancia materna es una de las formas más eficaces de asegurar la salud y la supervivencia de los niños. Se sabe que la leche materna es el mejor seguro de vida para los niños menores de 6 meses.

Sin embargo, los menores de 6 meses alimentados exclusivamente con leche materna no llegan al $40 \%$. Es por eso que la OMS asegura que un apoyo adecuado a las madres y a las familias para que inicien y mantengan la lactancia materna podría salvar la vida de muchos pequeños. Motivo por el cual, la OMS recomienda comenzar la lactancia en la primera hora de vida es decir, el contacto piel con piel con sus madres inmediatamente después del nacimiento sobre el abdomen y pecho materno, lo cual están alertas, estimulados por las caricias de sus madres. Este primer contacto de la mano o cabeza de los recién nacidos sobre el pecho, estimula la secreción de la oxitocina materna, comenzando así el flujo de leche, realzando las sensaciones de afecto para el recién nacido para luego iniciar la primera succión, siendo el efecto de sincronización de la primera mamada del recién nacido sobre la mortalidad infantil. Esta secuencia de eventos es importante para la supervivencia de los niños ${ }^{14}$. 
Sobre el conocimiento de la edad en que se debería brindar lactancia materna exclusiva, en esta investigación el mayor porcentaje de madres respondieron hasta los 6 meses de edad con el $70,8 \%$, como la misma OMS recomienda la lactancia materna exclusiva hasta alrededor de los seis meses de edad y la continuación de la misma, ya que reduce la frecuencia de infecciones gastrointestinales y respiratorias en lactantes, y se ha demostrado beneficios para su desarrollo y ningún déficit nutricional. La introducción gradual de los alimentos complementarios hasta el segundo año de vida y más, si fuera posible. Con el fin de ofrecer a sus hijos un crecimiento, desarrollo y salud óptimos. Posteriormente, hasta los dos años, los lactantes deben seguir con la lactancia materna complementaria con otros alimentos nutritivos 15 .

Sobre el conocimiento de la frecuencia de brindar lactancia materna, el 34,6\% respondieron que es a libre demanda y solo un $13,4 \%$ respondieron que se le debe dar cada 6 horas. La lactancia debe ser a libre demanda, implica ofrecer el pecho ante el primer indicio que él bebe quiere mamar, pero también respetar sino desea, es decir que no existe un horario para amamantar tanto de día como de noche, motivo por el cual se le debe amamantar cada vez y cuantas veces quiera; hay que olvidarse de los horarios y la duración de la toma. Cada bebé tiene su propio ritmo de lactar; la cantidad de leche será mayor cuantas más veces se le dé el pecho 16. Puesto que la glándula mamaria integrada por las acciones secretoras cumple dos funciones principales: la lactogénesis que es la producción de la leche materna, la cual está controlada por los estrógenos, prolactina. La capacidad funcional de las glándulas secretoras es el resultado de las concentraciones cambiantes de hormonas en el torrente sanguíneo de la madre, pero solo hasta que desaparecen las del embarazo, sobre todo la progesterona, que puede estimular a la prolactina, haciendo que la síntesis y la secreción de la leche se realicen en los alveolos, y la otra función es la de expulsión de la leche que se realiza mediante la oxitocina. Respecto al tiempo que debe durar una mamada, el $2.4 \%$ refieren que debe ser de 5 minutos y el $36.2 \%$ refieren que debe ser hasta que el niño ya no quiera mamar y quede satisfecho. Según el Dr. Mike Woolridge, refiere que esto es debido a la leche de inicio y del final de la mamada, es decir que la leche de inicio es alta en volumen y baja en grasas y calorías. A medida que la toma progresa, el contenido en grasa va aumentando y la leche correspondiente se parece más a la leche entera, hacia el final de la toma, la leche es más blanca que la del comienzo, debido a que es rica en grasas, lo cual hace que la leche sea un líquido rico en energía necesario para que el niño quede satisfecho y aumente de peso satisfactoriamente ${ }^{17}$, por lo que observamos que las madres están conscientes del tiempo para vaciar la fórmula láctea de los senos galactóforos, siendo necesario aun educar al respecto.

Sobre el tipo de alimentos que debe brindarle a su hijo, existe un mayor predominio con el $70,1 \%$, que solo leche materna debe brindar a su hijo, porque la leche materna contiene un $85-87 \%$ de agua, por lo que la madre lactante debe ingerir un volumen de líquido suficiente como para asegurar su producción de leche diaria y mantener un buen estado de hidratación. La leche materna siempre contiene agua suficiente para satisfacer la sed del bebé, con independencia que si la madre toma menos líquidos de los que realmente necesita. Sin embargo, no por ello la madre debe descuidar la ingesta abundante de líquidos, ya que de ello depende que mantenga un adecuado estado de hidratación, por ello los bebés que están bajo lactancia exclusivamente durante los primeros 6 meses de vida no necesitan beber agua en forma adicional, de lo contrario se corre el riesgo que contraiga diarreas $\mathrm{u}$ otras enfermedades ${ }^{18}$.

Por otro lado el conocimiento que tienen las madres acerca del tiempo suficiente que el niño debe lactar hasta que esté satisfecho, así como la separación espontánea del niño teniendo en cuenta la composición de la leche, la cual varía del principio al final de la tetada. Por esta razón, se 
recomienda que tomen el pecho el tiempo suficiente para ingerir la leche más nutritiva al inicio de la tetada quedando para el final menos grasa. Este análisis coincide con la hipótesis formulada anteriormente, que el nivel de conocimiento que tienen las madres primíparas sobre lactancia materna exclusiva era regular.

Respecto al nivel de conocimiento sobre la técnica de amamantamiento, hubo mayor predominio del $74.8 \%$, que el nivel de conocimiento fue buena, en segundo lugar con $22 \%$ fue regular y en tercer lugar con 3.2\% fue malo. La mujer por naturaleza experimenta el acto de amamantar a su niño, imitando de su alrededor y antepasados, según María Jesús Blázquez, cuando el niño succiona, se envía un mensaje al cerebro, este estimula la liberación de dos tipos de hormonas la prolactina que estimula la producción de la leche y la oxitocina, que favorece la salida de ésta ${ }^{19}$. El niño que coge todo el pezón hace que aumente la eficacia de la succión, esto es uno de los reflejos del niño que están ligado al instinto de la supervivencia humana, que se desarrolla y ejercita antes, durante y después del nacimiento, por lo tanto: a mayor succión habrá mayor producción de leche.

Referente al nivel de prácticas sobre la técnica de amamantamiento, tuvo un porcentaje del $43.3 \%$ que fue bueno, seguido de regular con $31.5 \%$, en tercer lugar muy bueno con $23.6 \%$ y una mínima de $1.6 \%$ que fue malo, esto se realizó por medio de la observación, evaluándose con el instrumento de la lista de cotejo, la cual constó de 5 criterios, cada uno se evaluaba con puntajes de $5,10,15$, 20 según la actuación de la madre primípara, valorizándose de la siguiente manera de 85-100, si la práctica de amamantamiento fue muy bueno, 70-84 si fue bueno, 55-69 si fue regular, 40-54 si fue malo y menos de 40 muy malo, obteniéndose un nivel de práctica de amamantamiento de bueno representando el $43.3 \%$ de las cifras relativas, las cuales realizan una buena técnica de amamantamiento, por la buena posición que adoptan tanto la madre como el niño que se mantuvieron cómodas, mediante la posición sentada que es la forma más fácil de aprender a dar de lactar, con los pies cómodos y el niño apoyado en almohadas. Según Tejerina, se debe mantener una postura correcta, tanto del niño como de la madre para que la madre se encuentre cómoda. El cuerpo del niño debe estar de cara a la madre y todo el cuerpo del recién nacido quedará alineado, evitando que el niño tenga que flexionar - rotar la cabeza, porque esto dificulta la succión 20.

Para garantizar una lactancia materna feliz, basta con utilizar una técnica adecuada, tener paciencia y no abandonar la lactancia ante la primera dificultad.

No se trata de que la mayoría de los niños tomen la mayor parte de la leche, sino de que todos los niños tomen toda la leche que necesiten, y eso sólo se consigue poniéndoles a mamar todo lo que quieran.

El lactante es el que debe determinar la duración de las tomas y no es posible establecer reglas arbitrarias. Únicamente que el dúo madre-hijo estén cómodos y estén controladas todas las posibles complicaciones de la técnica de lactancia y estaremos en la situación de lactancia exitosa.

\section{Conclusiones}

1. El nivel de conocimientos sobre lactancia materna exclusiva en madres primíparas es regular, equivalente a un $46.5 \%$, aceptándose la hipótesis formulada en el trabajo de investigación. El nivel de conocimientos sobre la técnica de amamantamiento fue buena con un porcentaje del $74.8 \%$, donde el mayor porcentaje de las madres primíparas que participaron son procedentes de la sierra que oscilan entre las edades de 19 a 25 años.

Madres que están en la etapa de adulto joven, que saben tomar decisiones, responsabilidades para optar por tan difícil tarea de ser padres y saber lo que más le beneficia para tener un niño muy saludable.

2. Acerca de la práctica de la técnica de amamantamiento en la madre primípara, 
corresponde a un resultado positivo, equivalente al 43.3\%, evaluado de bueno, motivo que la gran mayoría de las madres han tenido conocimientos de sus antepasados.

3. El mayor porcentaje de madres primíparas afirman que es importante la lactancia materna exclusiva, por todos los beneficios que ésta le ofrece al niño y la madre.

4. La lactancia materna exclusiva se brinda hasta los seis meses de edad, lo cual constituye una estrategia privilegiada para superar los problemas de desnutrición y morbimortalidad infantil.

\section{Bibliografía}

1. Pérez A, Pérez T, Petrucci D, Ramírez A, Roa J. Cumplimiento de Lactancia Materna Exclusiva y su Asociación con Algunas Enfermedades Infecciosas y Asma en Pacientes de 6 Meses 2 años del Ambulatorio Urbano Tipo II Barquisemeto - Estado Lara 2009. Universidad Centro Occidental Lizandro Alvarado. Pág. 9-10. Disponible en: Bibmed.ucla.edu.ve/DB/bmucla/edocs/texocompl eto/TEGWS125DV4C852009.pdf

2. Aguilar MI, Fernández M. Lactancia Materna Exclusiva. (Monografía internet). Departamento de Medicina Familiar, Facultad de Medicina: UNAM. (Citada enero 2013). Disponible en: www.ejournal.unam.mx/rfm/no50-

4/RFM050000406.pdf

3. Rioja S. Lactancia Materna Información para Amamantar. Consejería de Salud. Servicio de Promoción de la Salud. Gobierno de la Rioja. 2007. Disponible en: www.riojasalud.ed/fichero/lactanci a- castellano.pdf.

4. Lipa J. Factores Maternos y Laborales que intervienen en la Interrupción Temprana de la Lactancia Materna Exclusiva en Madres que acuden al consultorio de Crecimiento y Desarrollo del Centro Materno Infantil Tablada de Lurín. Junio 2008 (tesis para optar el título profesional de
Licenciada en Enfermería. Lima: Universidad Privada San Juan Bautista; 2009. Disponible en: www.alipso.com/. . /factores-maternoslaboralesque-intervienen

5. UNICEF, INEC. Análisis del Módulo de Lactancia Materna. Encuesta Nacional de Hogares 2010. Costa Rica 2011. Disponible en:

http//.www.ministeriodesalud.gob.cr/gestoresen salud / lactancia /artículosLac. 12122011 .pdf.

6. Osandon M, Llabaca J, Gajardo Ce, Castillo N, Namur L. Programa Iniciativa Hospital Amigo del Niño y la Madre. Fomento de la Lactancia Materna Programa Iniciativa Hospital Barros Luco Trudeau. Online. (Serie en internet). Chile; 2000. (Acceso 10 Octubre 2010). Vol. 71 (2): 98-106. Disponible en: http://www.scielo.cl/scielo.php?scripto $=$ sci_artte x\&ped.

7. Félix J. Martínez E y Sot e I o E. Prevalencia y Factores Biosociales Asociados al Abando investigación Científica en Ciencias de la Salud: Principios y Métodos. México: Mc Graw Hill, DF Interamericana.2000.

8. Instituto Nacional de Estadística e Informática Dirección Técnica de Demografía e Indicadores Sociales. Encuesta Demográfica y de Salud Familiar - ENDES, Indicadores de Resultado Identificado de Programas Estratégicos. Pág. 15. Perú. 2010. Disponible en: www1 .inei.gb.pe/biblioineipub/bancopub/Est/Lib 0859/libro.pdf.

9. Fondo de las Naciones Unidas para la Infancia. Situación de la Niñez en el Perú. Iniciativa de Inversión en la Infancia. 2012; Edición 82. Mayo 2012. (Acceso Noviembre 2012). Disponible en: www.inversionenlainfancia.net/web/blog/noticia/ 459

10. Instituto Nacional de Estadística e Informática - Encuesta Demográfica y de Salud Familiar. Indicadores de Resultados de los Programas Estratégicos. Lactancia Materna Exclusiva. Perú. Febrero 2011. (acceso Setiembre 2011). 
Disponible

en:

desa.inei.gob.pe/endes/investigaciones/libro_end es.pdf

11. Polit D, Hungler B. Investigación Científica en Ciencias de la Salud: Principios y Métodos. México: Mc Graw- Hill, DF Interamericana.2000.

12. García T. el cuestionario como instrumento de investigación. Etapas del proceso investigador: instrumentación. Almendralejo 2003. http://www.univsantana.com/sociologia/El_Cuest ionario.pdf

13. Semana Mundial de la Lactancia Materna: 1-7 de agosto. Confederación de Consumidores y Usuarios. Depto. Nutrición e Higiene Alimentaria. (acceso noviembre 2012). Disponible en: http:/ / www.cecu.es/noticias/Lactancia\%20matern a\%202009.pdf

14. Semana Mundial de la Lactancia Materna. La Lactancia Materna Durante la Primera Hora Salva un Millón de Bebes. 2007. Disponible en: http://www.redsalud.gov.cl/archivos/semanamun diallactancia.pdf

15. Nieto J. Guía de Lactancia Materna para Profesionales de la Salud. (Internet).Perú. Consejería de Salud del Gobierno de la Rioja. 2010. (acceso octubre 2011). Disponible en:

http://wwww.riojasalud.es/ficheros/af_protocolo_ lactancia.pdf
16. OMS, OPS, UNICEF. Consejería en Lactancia Materna: Curso de Capacitación. (internet). 1993. Estados Unidos de América. (acceso noviembre 2011). Disponible en:

http:/ /www.who.int/maternal_child_adolescent/d ocuments/pdfs/bc_participants_manual_es.pdf

17. Newman J. Guía de Lactancia Materna. Nuevo Comienzo. (Serie en internet). 2011 (citado Dic. 2012); volumen 15 (2): 2003.2011

18. Avalos M. Morbilidad de Lactante con lactancia Materna. En Municipio Cienfuegos EneroDiciembre 2006, Trabajo de Tesis para optar el título de Especialista de Primer Grado Materno Infantil. Cuba. (acceso agosto 2010). Disponible en:

http://www.sld.cu/galerias/pdf/sitios/enfermeria -pediatria/tesis_mariela.pdf

19. Blázquez M. Ventajas de la Lactancia Materna. Revista de Medicina Naturista.2000; $\mathrm{N}^{\circ} 1: 44-49$ I.S.B.N: 1476-3080, 2003, (acceso el 01 de Octubre del 2010. Disponible en:

http://www.unizar.es/mednaturista/lacta.

20. Tejerina A. Lactancia Materna, Centro de Salud Casona (acceso agosto 2009). Disponible en: http://www.down21.org/educpsc/alimentación/la ctancia 